\title{
Bubbles in Collision: Hydrodynamical Models
}

\author{
A.D. Chernin
}

Sternberg Astronomical Institute, Moscow University, 119899 Moscow, Russia

\begin{abstract}
Computer simulations and laboratory experiments with shock waves are suggested for the study of collisions of bubbles or superbubbles. They describe how the colliding bubbles merge with the formation of an expanding common shell and a complex internal dynamical structure which includes two vortex tori and collimated outflows.
\end{abstract}

\section{Introduction}

Expanding bubbles in the interstellar medium can come into contact and interact with each other. The major physical mechanism of this process is the interaction of expanding shocks. We study it with the use of the CourantFriedrichs analytical theory, new computer simulations and laboratory experiments with shock waves.

\section{The Courant-Friedrichs Configuration and Its Evolution}

The contact collision of shocks leads to the formation of a new regular dynamic structure in the interaction area studied in general terms by Courant and Friedrichs (1948). They analyzed a simple, but instructive example in which the fronts of the shocks are assumed to be spherical with equal radii. The structure includes the initial fronts, the annular Mach front, two tangential discontinuities and the reflected fronts. This configuration formed with these dynamical elements is not stationary and must evolve because of the interaction of these elements and their intrinsic transformations. As a result, the overall structure of the flow undergo considerable changes which, since a certain stage, can no longer be described by the Courant-Friedrichs theory.

With the same statement of the problem as in the Courant-Friedrichs picture, the supersonic collision of two expanding adiabatic shock fronts was studied in 3D (with axial symmetry) computer simulations (Voinovich and Chernin 1995). The method used in the computations was worked out at Ioffe Institute (Sankt Petersburg). The simulations give a picture of the advanced stages of the evolution of the Courant-Friedrichs structure.

The density of gas in the central area bounded by the Mach front and two reflected shocks increases ten times in the adiabatic regime. This dense and hot material forms a disc in the plane of symmetry of the flow. Later on the 


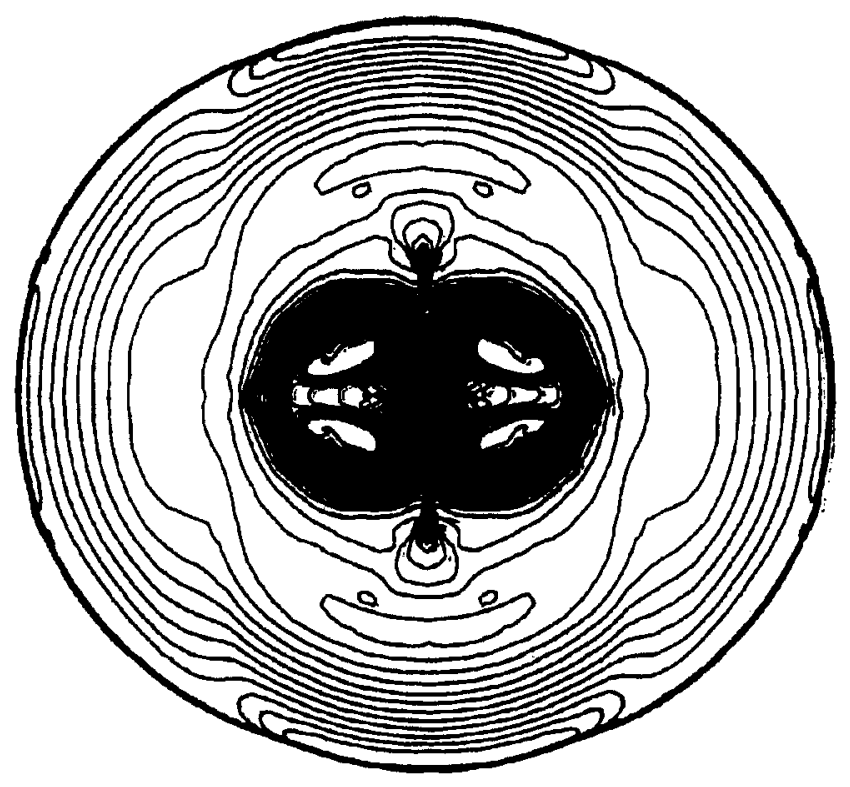

Fig. 1. Merging bubbles: the flow pattern indicated by density isolines.

disc transforms into a torus due to the gas outflows driven by the reflected shocks.

The simulations indicate also that the flows behind the reflected shocks move with increasing velocity when the shocks propagate into the area of the decreasing density in the cavities produced by the initial shocks. These flows reveal a tendency for a self-collimating behavior: while the density of the medium keeps decreasing along the direction of the shock propagation, the flows become more and more directed.

At the next stages, the secondary collimated outflows forms in the central area of the structure, and the central torus splits into two vortex tori, or toroidal vortexes. The vorticity here is mainly due to the transformation and decay of two tangential discontinuities of the Courant-Friedrichs structure.

In parallel with the evolution of their interaction area, two interacting bubbles as a whole merge, and the initial shocks together with the Mach front form a common envelope of more or less spherical shape which keeps expanding, as it is demonstrated by Fig.1. The high resolution structure of the central vortex tori and outflows from them at a late stage of the evolution is presented in Fig.2. 


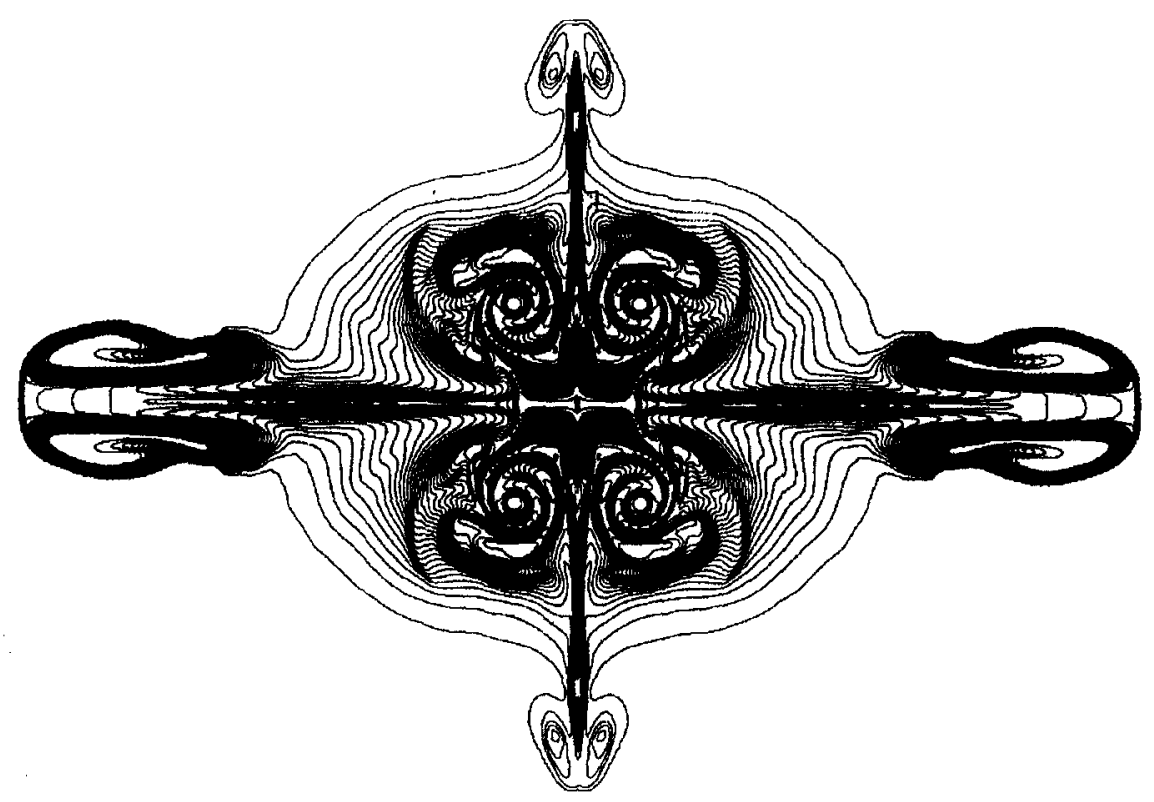

Fig, 2. The structure of the central tori and the outflows at a late stage of their evolution.

\section{Laboratory Experiments}

Can such a complex structure remain to be so regular and stable for long times? We have obtained a definite answer to this question from our laboratory experiments with shocks (Rybakov et al. 1997).

In the experiments, an adiabatic spherical shock front is produced by the explosion at the edge of a cooper needle under the action of a $80 \mathrm{~ns}$ long laser pulse of with an energy release of $12 \mathrm{~J}$. The front collides with a glass plate, and the reflected shock forms. Its dynamic is followed on a high speed camera. The duration of the experiment is about $1 \mathrm{~ms}$, and all the events are happening within a volume $5 \mathrm{~cm}$ across.

The structure and evolution of the reflected shock and the flow behind it are fairly similar, in general, to that in the Courant-Friedrichs theory and our computer simulations. It is most important that the experiments prove that the flow is stable, as a whole, against non-axisymmetric perturbations (which cannot be studied in the computer simulations because of the adopted symmetry). The picture of the flow keeps rather regular until its almost complete dissipation.

Unfortünately, we cannot control or even measure the strength of the 'initial' hydrodynamical perturbations that are present in the experiment. Perhaps it will be possible in a new set of experiments we plan now; if so, 
it would be interesting to compare these perturbations with what can be expected in real astrophysical situations.

\section{Conclusions}

The results described above provide, as we hope, new physical grounds for the studies of the collisions of expanding bubbles, their interaction and merging under the conditions when the process can be treated as adiabatic and radiative losses are not important. These conditions can be expected most commonly at early stages of the expansion of high-velocity shocks from supernova (see, for instance, Cox 1972, Lozinskaja 1986). Under these physical conditions, we can expect that the collisions and mergings of bubbles are accompanied by the formation of a complex and stable dynamical structure in the central area of the shock-shock interaction. It would be interesting to try to observe such structures - if not in the Local Interstellar Medium, then beyond it.

In the opposite extreme, when the cooling is very rapid, the collision of expanding shocks is almost completely inelastic, and the picture seems to be less dynamic: the merging bubbles produce a quasi-static central disc of increasing density with no prominent internal motions and an envelope formed by two initial expanding shock fronts without the Mach ring. The collisions of this type happen, perhaps, in the Large Magellanic Cloud where intersections of giant loops are seen (Meaburn 1980). This inelastic hydrodynamical process may be interesting for the physics of large-scale star formation observed in the regions like 30 Dor in the LMC (Chernin 1997).

Acknowledgements. I appreciate the kind support of the SOC.

\section{References}

Chernin A.D. (1997): in press

Courant R. \& Friedrichs K.O. (1948): Supersonic Flow and Shock Waves, N.Y., Interscience

Cox D.P. (1972): ApJ 178, 159

Lozinskaja T.A. (1986): Supernova and Stellar Wind, Moscow, Nauka Meaburn J. (1980): MNRAS 192, 365

Rybakov V.A., Artemiev V.I., Medvedyuk S.A., Voinovich P.A., \& Chernin A.D. (1997): in press

Voinovich P.A. \& Chernin A.D. (1995): Astr. Lett. 21, 926 